\title{
Ganciclovir intravítreo para retinite por citomegalovírus em pacientes com AIDS
}

Intravitreal ganciclovir for cytomegalovirus retinitis in patients with AIDS

\begin{tabular}{|c|c|}
\hline Áisa & idar ${ }^{1}$ \\
\hline Cristina & Muccioli ${ }^{2}$ \\
\hline Tércio & $\mathfrak{G u i a}^{3}$ \\
\hline tbens & Belfort \\
\hline
\end{tabular}

${ }^{1}$ Fellow do Setor de Uveítes e AIDS do Departamento de Oftalmologia da Universidade Federal de São Paulo Escola Paulista de Medicina.

${ }^{2}$ Chefe do Setor de Uveítes e AIDS do Departamento de Oftalmologia da Universidade Federal de São Paulo Escola Paulista de Medicina.

${ }^{3}$ Tecnólogo Chefe do Setor de Documentação Fotográfica da Universidade Federal de São Paulo - Escola Paulista de Medicina.

${ }^{4}$ Professor Titular do Departamento de Oftalmologia da Universidade Federal de São Paulo - Escola Paulista de Medicina.

Endereço para correspondência: Rua Botucatu, 822 São Paulo (SP) CEP 04023-062. E-mail: eyebr@web mail.epm.br

\section{RES UMO}

Objetivo: Avaliar a eficácia de alta dosagem de ganciclovir (2000 ou 4000 $\mu \mathrm{g}$ ) em pacientes com a síndrome da imunodeficiência adquirida (AIDS) para tratamento da retinite por citomegalovírus. Métodos: Estudo prospectivo para tratamento de retinite por citomegalovírus com injeção intravítrea de ganciclovir ( 2000 ou $4000 \mu \mathrm{g}$ ) em pacientes com diagnóstico de AIDS e que apresentavam intolerância ao tratamento com ganciclovir endovenoso. Este estudo foi realizado no Departamento de Oftalmologia da Universidade Federal de São Paulo - Escola Paulista de Medicina, no período de 1996 a 1998. Resultados: Dos 1950 pacientes examinados, 21 pacientes com diagnóstico de retinite por citomegalovírus e intolerância ao tratamento endovenoso foram tratados com injeções intravítreas de ganciclovir. A média de idade foi 37,1 anos e 31 olhos dos 21 pacientes receberam tratamento local. O comprometimento ocular era unilateral em 11 pacientes e bilateral em 10. Dezessete pacientes eram do sexo masculino. Dos 21 pacientes tratados, 11 pacientes (14 olhos) receberam injeção intravítrea na dosagem de $2000 \mu \mathrm{g}$ e 10 pacientes, 17 olhos, receberam injeções intravítreas na dosagem de $4000 \mu \mathrm{g}$. No grupo 1 (injeção intravítrea de $2000 \mu \mathrm{g}$ ) foi observado melhora da acuidade visual em $35,7 \%$ dos olhos, estabilidade da acuidade visual em $50 \%$ e piora em $14,3 \%$, sendo todos atribuídos a descolamento de retina. No grupo 2 (injeção intravítrea de $4000 \mu \mathrm{g}$ ) observamos melhora do quadro em $58,8 \%$, estabilidade do quadro em $23,5 \%$ e piora do quadro em $17,6 \%$ devido à catarata e descolamento de retina. Conclusão: Altas dosagens de ganciclovir intravítreo são eficazes para tratar a retinite por citomegalovírus. Os efeitos colaterais mais freqüentemente observados foram catarata e descolamento de retina e parecem não estar relacionados ao tratamento local.

Descritores: Síndrome de imunodeficiência adquirida; Retinite por citomegalovírus/ quimioterapia; Gangiclovir/uso terapêutico; Injeções; Corpo vítreo; Estudos prospectivos

\section{INTRODUÇÃO}

A retinite por citomegalovírus (CMV) é a infecção oportunista mais comum em pacientes com a síndrome da imunodeficiência adquirida (AIDS) ${ }^{(1-2)}$. Terapêutica antiviral com ganciclovir ${ }^{(3)}$ ou foscarnet $^{(4)}$ endove- $^{-}$ noso são eficazes no controle da progressão da retinite por CMV em pacientes com AIDS. Porém, o tratamento sistêmico pode produzir efeitos colaterais que levam a descontinuidade do tratamento. $\mathrm{O}$ tratamento local com injeções intravítreas de ganciclovir tem papel importante no controle terapêutico em pacientes com retinite por CMV os quais tiveram falha na 
terapia sistêmica. Os primeiros estudos disponíveis na literatura utilizaram dosagens de $200 \mu \mathrm{g}$ para o tratamento local da retinite por citomegalovírus. Posteriormente novos estudos foram realizados com doses maiores de ganciclovir e foi então observado que tal droga era eficaz e segura para o tratamento da retinite por citomegalovírus. Em publicações mais recentes, novas dosagens como 2000 e $4000 \mu \mathrm{g}$ relatam que o tratamento com altas dosagens de ganciclovir intravítreo não causam toxicidade à retina e são eficazes por períodos prolongados de tempo.

O objetivo deste trabalho é avaliar a eficácia, bem como observar as complicações relacionadas ao tratamento local com ganciclovir intravítreo (2000 e 4000 microgramas) em pacientes com retinite por CMV e AIDS e que apresentaram intolerância ao ganciclovir ou foscarnet endovenoso.

\section{MÉTODOS}

No período de 1996 a 1998 foram examinados 1950 pacientes infectados pelo vírus da imunodeficiência humana (AIDS) e $25 \%$ com retinite por citomegalovírus no ambulatório de uveítes e AIDS da Universidade Federal de São Paulo. Dos pacientes com retinite por citomegalovírus, 21 (31 olhos) apresentaram intolerância a medicação sistêmica, (ganciclovir ou foscarnet). Estes pacientes em estudo não estavam em tratamento com o HAART (Highly Active Anti-Retroviral Therapy).

O diagnóstico da infecção pelo HIV foi realizado pelo método de ELISA (enzyme linked immunosorbent assay), e confirmado pelo Western blot. O diagnóstico de retinite por citomegalovírus foi realizado baseado no aspecto clínico da lesão e exclusão de outros diagnósticos diferenciais.

Os pacientes foram submetidos a exame oftalmológico que constou de medida da acuidade visual, biomicroscopia, tonometria de aplanação e oftalmoscopia binocular indireta. Retinografia, angiofluoresceinografia e indocianina verde foram realizados quando indicados.

As lesões foram localizadas na retina conforme o esquema de Holland et al.${ }^{(5)}$ que dividiram a retina em zona I ( 2 diâmetros papilares a partir do centro da papila e 3 diâmetros papilares a partir da mácula), zona II (zona I até as veias vorticosas) e zona III (zona II até ora serrata).

As lesões foram classificadas em 3 formas clínicas (hemorrágica, granular e de "vasos congelados"). O primeiro, clássico, chamado de típico ou hemorrágico, é caracterizado por áreas retinianas esbranquiçadas e áreas hemorrágicas, geralmente próximas às arcadas vasculares ou ao nervo óptico, e com bordas irregulares. O segundo tipo de retinite é o granular ou atípico em que há infiltrados focais granulares. E o terceiro tipo caracteriza-se por uma vasculite severa com artérias e veias mostrando intenso embainhamento, lembrando o aspecto de "vasos congelados", podendo ou não estar associado à forma clássica.

Os critérios de inclusão foram: 1) paciente com diagnóstico de AIDS e maior de 18 anos; 2) diagnóstico de retinite por
CMV; 3) paciente que já teve 1 ou mais tratamentos de indução com dose de ataque com ganciclovir ou foscarnet; 4) paciente com mais de $25 \%$ da retina envolvida pela retinite por CMV; 6) assinatura do documento de consentimento.

Os critérios de exclusão foram: 1) infecção ocular externa no olho a ser tratado; 2) associação de outras infecções oculares como: retinite herpética, retinocoroidite por toxoplasmose, outras doenças que impediriam a avaliação da retinite por CMV; 3) alteração ocular que impedia a observação das estruturas oculares posteriores do olho a ser tratado; 4) descolamento de retina; 5) implantes de ganciclovir para tratamento para retinite por CMV; 6) pacientes alérgicos ou história de intolerância ao ganciclovir; 7) óleo de silicone no olho a ser tratado; 8) diagnóstico de pseudo retinite pigmentosa; 9) história de sífilis em curso; 10) terapia sistêmica com ganciclovir oral; 11) paciente grávida.

Os pacientes foram randomizados para dois grupos de diferentes dosagens ( $2000 \mu \mathrm{g}$ ou $4000 \mu \mathrm{g}$ de ganciclovir intravítreo). O primeiro grupo de pacientes envolvidos no estudo recebeu $2000 \mu \mathrm{g}$ e o segundo $4000 \mu \mathrm{g}$ de ganciclovir intravítreo. Isso foi realizado por sorteio. Os pacientes receberam injeções intravítreas semanais de ganciclovir por 3 semanas no período de indução (ou inicial); e na fase de manutenção foram realizadas injeções intravítreas de ganciclovir 1x/semana. A manutenção foi realizada em média por 22 semanas.

Se o segundo olho viesse a desenvolver retinite por citomegalovírus ativa, seria oferecido a escolha de receber a medicação também neste olho.

A técnica utilizada foi injeção intravítrea usando seringa de insulina, aplicação a $4 \mathrm{~mm}$ do limbo na região temporal superior de 2000 ou 4000 microgramas em $0,1 \mathrm{ml}$ dependendo do grupo envolvido. Antes desse procedimento era realizada assepsia e anestesia local com anestésicos tópicos com a finalidade de diminuir a dor de qualquer injeção.

Dos 1950 pacientes examinados, $21(1,07 \%)$ apresentaram diagnóstico de retinite por $\mathrm{CMV}$ e intolerância à medicação sistêmica (ganciclovir ou foscarnet). Foram submetidos à injeção intravítrea de ganciclovir, 31 olhos de 21 pacientes. Onze pacientes (14 olhos) foram tratados com dosagem de $2000 \mu \mathrm{g}$ e 10 pacientes ( 17 olhos) com dosagem de $4000 \mu \mathrm{g}$ conforme tabela 2.

\section{RESULTADOS}

Dos 21 pacientes examinados, 17 (81\%) eram do sexo masculino e o acometimento era unilateral em $11(52,4 \%)$ e bilateral em $10(47,6 \%)$. A média de idade foi de 37,1 anos (variando de 30 a 43 anos). Os fatores de risco para infecção pelo HIV foram: contato homossexual em 10 (47,60\%) pacientes; contato heterossexual em $9(42,90 \%)$ e uso de drogas endovenosas em $2(9,50 \%)$ conforme demonstra a tabela 1 .

No início do tratamento os pacientes apresentavam baixa de acuidade visual conforme demonstra a tabela 2 . As lesões estavam localizadas nas zonas 1 e 2 em 3 olhos (14,5\%), nas 
zonas 1, 2, 3 em 11 olhos (47,6\%), e nas zonas 2, 3 em 17 olhos $(80,9 \%)$.

\begin{tabular}{|c|c|c|c|}
\hline \multicolumn{4}{|c|}{ Tabela 1. Aspectos demográficos } \\
\hline Dos & se $2000 \mu g$ & Dose $4000 \mu g$ & Total \\
\hline$N^{\circ}$ de pacientes & 11 & 10 & 21 \\
\hline $\begin{array}{l}\text { - Sexo } \\
\text { - Masculino } \\
\text { - Feminino }\end{array}$ & $\begin{array}{l}08 \\
03\end{array}$ & $\begin{array}{l}09 \\
01\end{array}$ & $\begin{array}{l}17 \\
04\end{array}$ \\
\hline $\begin{array}{l}\text { - Número de olhos envolvidos } \\
\text { - Unilateral } \\
\text { - Bilateral }\end{array}$ & $\begin{array}{l}14 \\
08 \\
03\end{array}$ & $\begin{array}{l}17 \\
03 \\
07\end{array}$ & $\begin{array}{l}31 \\
11 \\
10\end{array}$ \\
\hline $\begin{array}{l}\text { - Idade (anos) } \\
\text { - Média } \\
\text { - Intervalo }\end{array}$ & $\begin{array}{l}38,09 \\
33-43\end{array}$ & $\begin{array}{c}36,1 \\
30-42\end{array}$ & $\begin{array}{c}37,1 \\
30-43\end{array}$ \\
\hline $\begin{array}{l}\text { - Transmissão HIV } \\
\text { - Contato Homossexual } \\
\text { - Contato Heterossexual } \\
\text { - Uso de drogas endovenosas }\end{array}$ & $\begin{array}{l}07 \\
04\end{array}$ & $\begin{array}{l}03 \\
05 \\
02\end{array}$ & $\begin{array}{l}10 \\
09 \\
02\end{array}$ \\
\hline
\end{tabular}

No grupo I (injeção intravítrea de $2000 \mu \mathrm{g}$ ) observamos melhora da acuidade visual em 5 (35,8\%) olhos, estabilidade da visão em 7 (50\%) olhos e piora em 2 olhos devido ao descolamento de retina. No grupo II (injeção intravítrea de $4000 \mu \mathrm{g})$ observamos melhora da acuidade visual em 10 olhos $(58,8 \%)$, estabilidade em 4 olhos $(23,5 \%)$ e piora em 3 olhos $(17,6 \%)$ que apresentaram catarata associada ao descolamento de retina conforme demonstram as tabelas 1 e 2 .

Com relação à forma clínica das lesões observamos no grupo I (14 olhos) a forma clássica em 4 (28,5\%) olhos; a forma granular em 8 olhos $(57,14 \%)$ e o aspecto de vasos congelados em $2(14,8 \%)$ olhos. Após o tratamento observou-se melhora do aspecto clínico em $35,8 \%$, cicatrização acompanhada de necrose retiniana em $50 \%$ e em $14,2 \%$ o descolamento de retina.

No grupo II (17 olhos) observamos a forma clássica em 5 $(29,4 \%)$ olhos, a forma granular em $10(58,8 \%)$ olhos, e o aspecto de vasos congelados em $2(11,7 \%)$ olhos. Após o

\begin{tabular}{|c|c|c|c|c|c|c|c|}
\hline & \multicolumn{5}{|c|}{$\begin{array}{l}\text { Tabela } 2 . \\
\end{array}$} & & \\
\hline & Sexo & Olho & Idade & Grupo Risco & AV Inicial & Av Final & Complicações \\
\hline 1 & M & OE & 35 & $\mathrm{HE}$ & $20 / 30$ & $20 / 20$ & \\
\hline 2 & M & OE & 36 & $\mathrm{HE}$ & $20 / 25$ & $20 / 25$ & \\
\hline 3 & M & OD & 38 & $\mathrm{HO}$ & $\mathrm{MM}$ a $1 \mathrm{~m}$ & PL & Descolamento de retina \\
\hline 4 & M & OE & 41 & $\mathrm{HO}$ & $20 / 40$ & $20 / 30$ & \\
\hline 5 & M & OE & 38 & $\mathrm{HO}$ & $\mathrm{CD}$ a $1 \mathrm{~m}$ & $\mathrm{CD}$ a $1 \mathrm{~m}$ & \\
\hline 6 & $\mathrm{~F}$ & $\mathrm{AO}$ & 42 & $\mathrm{HE}$ & $\begin{array}{l}20 / 40 \\
20 / 25\end{array}$ & $\begin{array}{l}20 / 30 \\
20 / 25\end{array}$ & \\
\hline 7 & $\mathrm{~F}$ & OE & 43 & $\mathrm{HO}$ & $\mathrm{CD}$ a $1 \mathrm{~m}$ & PL & Descolamento de retina \\
\hline 8 & $\mathrm{~F}$ & OD & 39 & HE & $\mathrm{CD}$ a $1 \mathrm{~m}$ & $\mathrm{CD}$ a $1 \mathrm{~m}$ & \\
\hline 9 & M & OE & 36 & $\mathrm{HO}$ & $20 / 30$ & $20 / 30$ & \\
\hline 10 & M & $\mathrm{AO}$ & 38 & $\mathrm{HO}$ & $\begin{array}{l}20 / 40 \\
20 / 50\end{array}$ & $\begin{array}{l}20 / 40 \\
20 / 50\end{array}$ & \\
\hline 11 & M & $\mathrm{AO}$ & 33 & $\mathrm{HO}$ & $\begin{array}{l}20 / 40 \\
20 / 60\end{array}$ & $\begin{array}{l}20 / 30 \\
20 / 40\end{array}$ & \\
\hline \multicolumn{8}{|c|}{ Grupo II - Dosagem $=4000 \mu g$} \\
\hline & Sexo & Olho & Idade & Grupo Risco & Av Inicial & AV Final & Complicações \\
\hline 1 & M & $\mathrm{AO}$ & 42 & DEV & $\begin{array}{c}20 / 200 \\
20 / 80\end{array}$ & $\begin{array}{l}20 / 25 \\
20 / 30\end{array}$ & \\
\hline 2 & M & $\mathrm{AO}$ & 36 & $\mathrm{HE}$ & $\begin{array}{l}20 / 60 \\
20 / 40\end{array}$ & $\begin{array}{c}20 / 200 \\
20 / 25\end{array}$ & Catarata \\
\hline 3 & M & OD & 34 & $\mathrm{HO}$ & $C D$ a $50 \mathrm{~cm}$ & $20 / 200$ & \\
\hline 4 & M & $\mathrm{AO}$ & 36 & $\mathrm{HO}$ & $\begin{array}{l}20 / 30 \\
20 / 30\end{array}$ & $\begin{array}{l}20 / 25 \\
20 / 25\end{array}$ & \\
\hline 5 & M & OD & 36 & $\mathrm{HE}$ & $20 / 60$ & $20 / 60$ & \\
\hline 6 & M & $\mathrm{AO}$ & 35 & $\mathrm{HO}$ & $\begin{array}{l}20 / 25 \\
20 / 25\end{array}$ & $\begin{array}{l}20 / 25 \\
20 / 25\end{array}$ & \\
\hline 7 & M & OE & 30 & DEV & MM & $\mathrm{CD}$ a $1 \mathrm{~m}$ & \\
\hline 8 & $\mathrm{~F}$ & $\mathrm{AO}$ & 30 & $\mathrm{HE}$ & $\begin{array}{l}\mathrm{CD} \text { a } 1 \mathrm{~m} \\
\mathrm{CD} \text { a } 4 \mathrm{~m}\end{array}$ & $\begin{array}{l}20 / 200 \\
20 / 400\end{array}$ & \\
\hline 9 & M & $\mathrm{AO}$ & 41 & $\mathrm{HE}$ & $\begin{array}{l}\mathrm{CD} \text { a } 1 \mathrm{~m} \\
\mathrm{CD} \text { a } 1 \mathrm{~m}\end{array}$ & $\begin{array}{l}\text { CD a } 1 \mathrm{~m} \\
20 / 100\end{array}$ & \\
\hline 10 & $M$ & $\mathrm{AO}$ & 41 & $\mathrm{HE}$ & $\begin{array}{c}\mathrm{CD} \text { a } 1 \mathrm{~m} \\
\mathrm{PL}\end{array}$ & $\begin{array}{l}\mathrm{PL} \\
\mathrm{SPL}\end{array}$ & $\begin{array}{c}\text { Catarata } \\
\text { Descolamento de retina }\end{array}$ \\
\hline
\end{tabular}


tratamento observamos regressão das lesões com melhora do aspecto clínico em $59 \%$, necrose retiniana em $23,5 \%$ e em $17,5 \%$ observou-se o aparecimento de descolamento de retina e catarata.

Não foram observadas doenças oportunistas no período de estudo desses pacientes.

\section{DISCUSSÃO}

A retinite por citomegalovírus é a mais importante complicação ocular da $\operatorname{AIDS}^{(1-2)}$. O tratamento endovenoso é eficaz no tratamento de retinite por CMV, porém freqüentemente há necessidade de descontinuidade do tratamento EV em conseqüência dos efeitos colaterais ${ }^{(3)} \mathrm{e}$, embora estudos prévios tenham confirmado a eficácia do ganciclovir intravítreo, este tem sido reservado para pacientes que apresentam intolerância à terapia sistêmica. Heineman e cols. ${ }^{(6)} \mathrm{em} 1989$ estudaram a eficácia do ganciclovir intravítreo na dosagem de 200 microgramas em 7 pacientes ( 14 olhos) por uma média de 235 semanas. Observou que todos os pacientes responderam a terapia inicial, enquanto que 6 olhos controles não tratados demonstraram progressão da retinite por citomegalovírus. Endoftalmite por estafilococos epidermides foi observado em $7 \%$, dado este que não foi observado em nosso estudo.

Hodge e cols. ${ }^{(7)}$ em 1996 estudaram a eficácia do ganciclovir intravítreo em 40 pacientes com retinite por citomegalovírus na dosagem de 400 microgramas. Quinze pacientes $(37,5 \%)$ tiveram 19 novas infecções oportunistas durante o período observado mas nenhum desenvolveu CMV sistêmico. No presente estudo observamos tanto no grupo I, como no grupo II, infecções oportunistas em $18 \%$ e $20 \%$ dos pacientes, respectivamente. Isso pode ser explicado pelo uso de novas drogas antiretrovirais. Hodge e cols. ainda observaram retinite aguda em 48,4\% dos olhos enquanto recebiam a dose de manutenção com uma média de tempo de progressão de 14,7 semanas. Retinite por citomegalovírus ocorreu em 30,4\% dos olhos envolvidos num seguimento médio de 3,1 anos. Endoftalmite bacteriana foi uma complicação do tratamento em $2,5 \%$ dos olhos, e descolamento de retina em 12,5\% dos olhos. Porcentagem esta menor que a encontrada no nosso estudo que observou descolamento de retina no grupo I em $18 \%$ e no grupo II em $20 \%$.

Young e cols. ${ }^{(8)}$ em 1998 estudaram 42 pacientes ( 74 olhos) tratados com injeções intravítreas na dosagem de 2000 microgramas e 18 pacientes ( 27 olhos) tratados com ganciclovir endovenoso. No grupo das injeções intravítreas, a acuidade visual era estabilizada em $86,5 \%$. Dado este semelhante do grupo I do nosso estudo, onde observamos estabilidade da acuidade visual em $85,8 \%$ considerando os casos de melhora.

Hodge e cols. ${ }^{(7)} \mathrm{em} 1997$ estudaram 14 pacientes (16 olhos) tratados com injeções intravítreas na dosagem de $4.000 \mathrm{mi}-$ crogramas. O edema macular cistóide foi observado em 3 (21.4\%) pacientes complicação não observada em nosso estudo nos grupo I e II.
Devido ao descolamento de retina ser observado em 17 a $34 \%$ dos pacientes com retinite por citomegalovírus acreditamos, que os casos observados em nosso estudo não sejam devido às injeções intravítreas.

\section{CONCLUSÃO}

Concluímos que as injeções intravítreas de ganciclovir são eficazes para o tratamento da retinite por CMV, controle de recidivas e tem a grande vantagem de não apresentar efeitos colaterais sistêmicos ou locais.

ABS TRACT

Purpose: To study high intravitreal dosage of ganciclovir (2000 or 4000 micrograms) in acquired immunodeficiency syndrome (AIDS) and cytomegalovirus retinitis. Methods: Prospective study for high dose intravitreal ganciclovir (2000 or 4000 micrograms) between 1996 and 1998 at the Federal University of São Paulo - Paulista Medical School in patients with diagnosis of AIDS, herpetic cytomegalovirus and intolerance to intravenous or oral treatment. Results: Of 1950 patients examined, $21(1.07 \%)$ were intolerant to systemic treatment (ganciclovir or foscarnet). Of those, 21 with 37.1 years average age, corresponding to 31 eyes were treated with intravitreous medication. Seventeen patients $(81 \%)$ were male. Of the 21 patients, 11 (14 eyes) received $2000 \mu \mathrm{g}$ intravitreous medication and 10 (17 eyes) received $4000 \mu \mathrm{g}$. In the beginning of the treatment $100 \%$ of the patients showed low vision. At the end of the treatment ( 22 weeks on average), group I (intravitreous medication $2000 \mu \mathrm{g}$ ) showed increase of visual acuity (VA) in $35.7 \%$, the same VA in $50 \%$ and decrease of VA in $14.3 \%$ due to retinal detachment. In the group II (intravitreous medication $4000 \mu \mathrm{g}) 58.8 \%$ had increased VA, $23.5 \%$ had the same VA and $17.6 \%$ had decrease of VA due to cataract and retinal detachment. Conclusions: Intravitreous injection of a high dose (2000 or 4000 micrograms $/ 0,1 \mathrm{ml}$ ) of ganciclovir for the treatment of a CMV retinitis is satisfactory. The adverse effects are cataract and retinal detachment. The retinal detachment could not be conclusively attributed to the injections and was probably a secondary complication of cytomegalovirus retinitis.

Keywords: Acquired immunodeficiency syndrome; Cytomegalovirus retinitis/ drug therapy; Ganciclovir/therapeutic use; Injections; Vitreous body; Prospective studies

\section{REFERENCIAS}

1. Pepose JS, Holland GN, Neston MS, Cochran AJ, Foos RY. Acquired Immunodeficiency Syndrome: pathogenic mechanisms of ocular disease Ophthalmology 1985;92:472-84. 
2. Muccioli C, Belfort Jr. R, Lottemberg C, Lima J, Santos P, Kim M, et al. Achados oftalmológicos em AIDS: avaliação de 445 casos atendidos em um ano. Rev Assoc Med Bras 1994;40:155-8.

3. Holland GN, William CB, Mastre BA, Kaplan HJ. Controlled retrospective study of ganciclovir treatment for cytomegalovirus retinopathy. Use of a standardized system for the assessment of disease outcome. Arch Ophthalmol 1989;107:1759-66

4. Palestine AG, Polis MA, De Smet MD, Baird BF, Falloon J, Kovacs JA, et al. A randomized controlled trial of foscarnet in the treatment of cytomegalovirus retinitis in patients with AIDS. [commented on Ann Intern Med 1992;116:604-5]. Ann Intern Med 1992;115:665-73.
5. Cantrill HL, Henry K, Melrol NH, Knobloch WH, Ramsay R C, Balfgur $\mathrm{HH}$. Treatment of cytomegalovirus retinitis with intravitreal ganciclovir. Ophthalmology 1989;96:367-74.

6. Heinemann MH. Long term intravitreal ganciclovir therapy for cytomegalovirus retinopathy. Arch Ophthalmol 1989;107:1767-72.

7. Hodge WG, Lalonde R G, Sampalis J, Deschênes J. Once weekly intraocular injections of ganciclovir for maintenance therapy of cytomegalovirus retinitis: clinical and ocular outcome. J Infect Dis 1996;174:393-6.

8. Young S, Morlet N, Besen G, Wiley CA, Jones P, Gold J, et al. High dose (2000 micrograms) intravitreous ganciclovir in the treatment of cytomegalovirus retinitis. Ophthalmology 1998;105:1404-10.

\title{
II CONGRESSO DA SBAO SOCIEDADE BRASILEIRA DE ADMINISTRAC̣ÃO EM OFTALMOLOCIA
}

\author{
06 a 08 Abril/2002
}

\section{Hotel Transamérica São Paulo}

Informac̣ões/Inscric̣ões:

- JDE - Tels: 05511 287-6999 / 287-8109

Fax: $05511288-8157$

e-mail: jdecomev@.vol.com.br

- SBAO - Tel/Fax: $055113266-4538$

e-mail: sbao@sbao.com.br

Workshop - Tribuna Livre

Médicos - Administradores - Pessoal de Apoio 